\title{
DESIGN, FABRICATION, AND TEST OF A 5-CM APERTURE, 1-M LONG SUPERCONDUCTING DIPOLE PROTOTYPE FOR HIGH ENERGY HADRON COLLIDER*
}

\author{
A. Devred \\ Superconducting Super Collider Laboratory ${ }^{\dagger}$ \\ 2550 Beckleymeade Ave. \\ Dallas, TX 75237 \\ September 1990
}

\begin{abstract}
DISCLAIMER
This report was prepared as an account of work sponsored by an agency of the United States Government. Neither the United States Government nor any agency thereof, nor any of their employees, makes any warranty, express or implied, or assumes any legal liability or responsibility for the accuracy, completeness, or usefulness of any information, apparatus, product, or process disclosed, or represents that its use would not infringe privately owned rights. Reference herein to any specific commercial product, process, or service by trade name, trademark, manufact'rer, or otherwise does not necessarily constitute or imply its endorsement, recommendation, or favoring by the United States Government or any agency thereof. The views and opinions of authors expressed herein do not necessarily state or reflect those of the United States Government or any agency thereof.
\end{abstract}

"Presented at the Applied Superconductivity Conference, Snowmass, Colorado, September 24-28, 1990.

† Operated by the Universities Research Association, Inc., for the U.S. Department of Energy under Contract No. DE-AC02-89ER40486. 


\section{DESIGN, FABRICATION, AND TEST OF A 5-CM APERTURE, 1-M LONG SUPLRCONDUCTING DIPOLE PROTOTYTE FOR HHGH ENERGY HADRON COLLIDER}

T. Shintomi, T. Ogitsu, A. Terashima, K. Maehata, M. Wake," and H. Hirabayashi KEK, National Laboratory for High Energy Physics,

1-1 Oho, Tsukuba-shi lbaraki-ken 305 Japan

\section{A. Devred}

KEK, National Laboratory

for High Energy Physics, Japan \& SSC Laboratory ${ }^{\dagger}$

2550 Beckleymeade Avenue

Dallas, TX 75237 USA

\section{Abstract}

A $1-\mathrm{m}$ long superconducting dipole prototype with an aperture of $5 \mathrm{~cm}$ and a rated field of $6.6 \mathrm{~T}$ was built and tested. This model was hased on a two-layer cosine-theta coil clamped by stainless steel collars inside a laminated iron yoke, with a large keystone-angle cable and no wedge. The cold mass was encased in an outer stainless steel skin. The magnet was instrumented with voltage taps, which allow the location of the quench start, and with strain gauges, which allow the measurement of the coil stress variations during assembly, cool-down, and energization. Prior to the assembly, several tests were carried out in order to understand the mechanical properties of the coil and 10 determine a proper calibration for the strain gauges. This paper reports these design studies, with emphasis on the calibration problem, followed by a discussion of the magnet assembly and quench performance in light of the mechanical measurements.

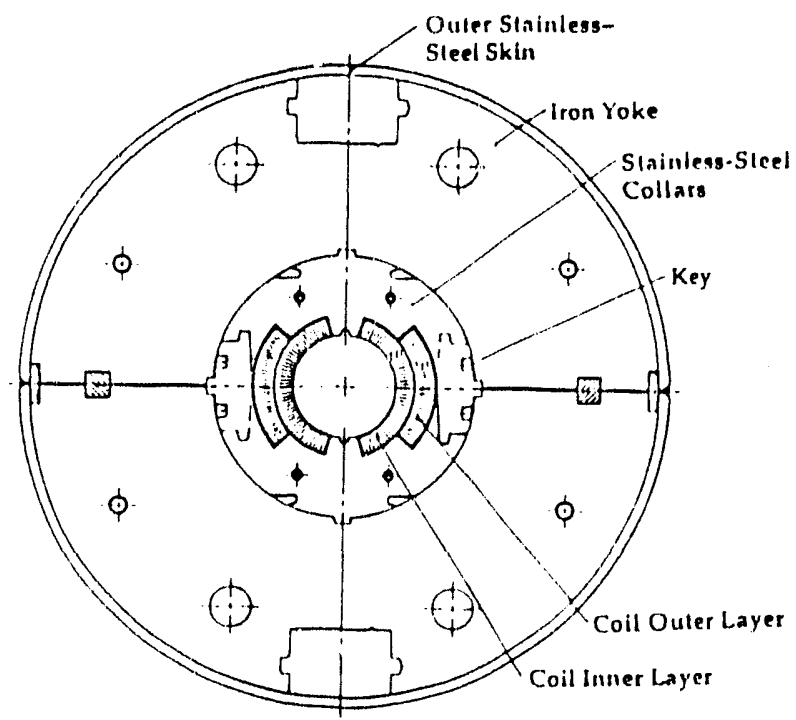

Figure 1: Cross-sectionnal view of magnet SD501/02 cold mass.

\footnotetext{
- Now at Departicent of Nuclear Engineering, Kyushu University, Hakozaki, Higashi-ku. Fukuoka-shi 812, Japan.

" Now at Fermi National Laboratory, Batavia, IL 60510, USA.

+ Operated by the Universitics Rescarch Association, Inc., for the United States Department of Encrgy.
}

\section{Introduction}

Several high energy particle accelerators projects, like the Superconducting Super Collider (SSC) in the US $\Lambda^{1}$ or the Large Hadron Collider (LHC) in Europe, ${ }^{2}$ call for the development of small-aperture, high-field superconducting dipoles: $5 \mathrm{~cm}$ and $6.6 \mathrm{~T}$ for the SSC, $5 \mathrm{~cm}$ and $10 \mathrm{~T}$ for the LHC. The present designs for both the SSC and LHC dipole coils rely on a cosinetheta distnbution of conductors, as was the case for the Tevatron and the Hera dipoles. However, because of the smaller aperture, the conductor-keystone angle has to be larger. Keystoning is of course a delicate operation for there are risks of degrading the conductor critical-current density, especially at the thin edge. The authors of the 1986 conceptual design report of the SSC $\mathrm{S}^{1}$ therefore adopted a conservative allitude and selected a $1.6^{\circ}$ keystone-angle for the inner-layer conductor (the conductor positions in the cross-section are adjusted by mean of asymmetric wedges). Since then, an important $R \& D$ effort has been carried out in industry in order to understand the cabling degradation and to limit its effects. 3,4 it was found that the creation of a copper sheath at the periphery of the conductor strands could help protect the superconducting filaments during cabling and reduce the degradation to an acceptable level. Large keystone-angle cables ( $3^{\circ}$ or more) wilh little degradation (5\% or less) are now available, rendering possible the study of wedgeless design for the SSC and LHC dipoles.

In 1989, KEK built a first $5-\mathrm{cm}$ aperture, $1-\mathrm{m}$ long wedgeless dipole prototype, SD501/01, which used a $3.07^{\circ}$ keystone-angle cable. Details on the features of this magnet and partial test results can be found elsewhere. 5,6 Except for the iwo-layer coil, the design concepts were very lose to that of the present SSC design: 7,8 the coil was clamped into laminated stainless.steel collars, iron yoke laminations were located outside the collars to enhance the magnetic field, and the cold mass was encased in an outer stainless steel skin, delimiting the region where the helium circulates. Initially, the magnet exhibited poor training behavior and required eight quenches to reach the design current. This was attributed to low precompression in the coil (precompression is what results from the squeezing of the coil into the collars at room temperature). It was later disassembled and reassembled with a higher precompression; the training was then reduced, requiring only two quenches to reach the design current. No particular problens were found in relation to the conductor. This first prototype thus demonstrated the feasibility of the wedgeless concept with a large keystone-angle cable. On the other hand, the disassembly/reassembly experiment pointed out the importance of coil precompression on the training behavior. A literature review ${ }^{9}$ revealed that there were very few comprehensive studies of the mechanical properties of the coil. Therefore, it seemed appropriate to undertake a program aimed at understanding these properties and their relation to training. 
This paper presents the design studies, assembly data, and lest results of the second $5-\mathrm{cm}$ aperture, $1-\mathrm{m}$ long wedgeless dipole prototype built at KEK, SD 01/02. Figure 1 shows a croossectional view of the cold mass, the parameters of which are summarized in table 1. The conductor parameters are presented in table 2 . The conductor insulation consists of two lay. ers of Kapton; the first one, $25-\mu \mathrm{m}$ thick, is helically wrapped with a $50 \%$ overlap, the second one, $50 . \mu \mathrm{m}$ thick, is wrapped with a 1 -mm gap and is covered with $25 \mu \mathrm{m}$ of B-stage epoxy on its outer surface. The main differences between SD501/02 and SD501/01 are the inner- and outer-layer cable widths, which were increased from $9.3109 .7 \mathrm{~mm}$ lo allow more operating margin. In order to understand training behavior, the magnet was equipped with a total of 39 voltage taps on the coil inner layer to locate the quench origins, ${ }^{10}$ and 8 beam-type strain gauge transducers to measure the azimuthal stress exerted by the coil against the collar pole." Prior to the assembly, the strain gauges were calibrated on conductor stacks representative of the coil inner and outer layers, at both room and liquid $\mathrm{ni}$ trogen temperatures, and a certain number of tests were carried out to understand the mechanical properties of the stacks. We shall first report on these experiments. We shall then present the mechanical data recorded during the magnet assembly and testing and discuss the quench performance.

Table 1. Selected Parameters of Magnet SD501/02

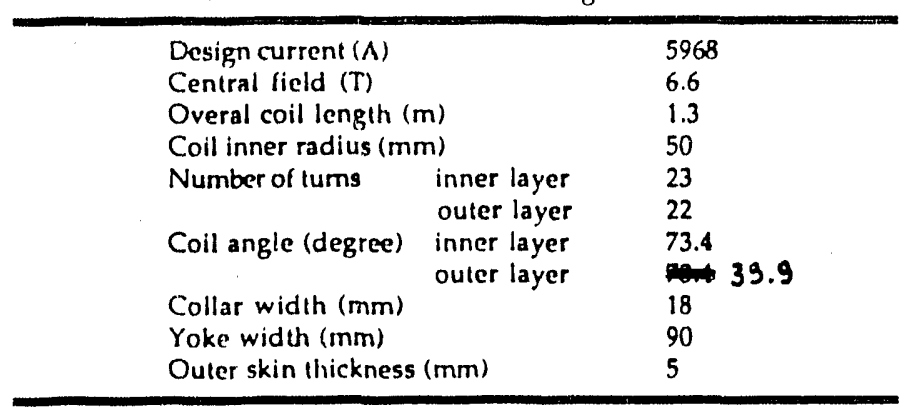

Table 2. Selected Paramelers of Magnet SD501/02 Conductors

\begin{tabular}{lll} 
& & \\
& Inner & Outer \\
\hline Cable width $(\mathrm{mm})$ & 9.7 & 9.688 \\
Cable mid-thickness $(\mathrm{mm})$ & 1.472 & 1.078 \\
Keystone angle $($ degrce) & 3.204 & 1.813 \\
Twist pitch $(\mathrm{mm})$ & 80.0 & 74.5 \\
Number of strands & 23 & 30 \\
Strand diameter $(\mathrm{mm})$ & 0.847 & 0.650 \\
Fiiament diamcter $(\mu \mathrm{m})$ & 6.3 & 6.1 \\
Copper to niobium-titanium ratio & $1.2 / 1$ & $1.7 / 1$ \\
Copper RRR & 142 & 155 \\
Critical current at 5 T and 4.2 K $(\Lambda)$ & 14500 & 9900 \\
\hline
\end{tabular}

\section{Study of Coil Propertics and Strain Gauge Calibration}

The beam-lype strain gauge transducers used in SD501/02 are of a similar design than that developed by BNL., except that two strain gauges are mounted on each beam in a Poisson halfbridge configuration. This configuration is thought to provide better compensation for thermal and magneto-resistive effects. It also reduces the number of wires.

The conductor stack representative of the coil inner layer, which was used for the calibration, consists of 24 insulated conductors covered with two $125 \cdot \mu \mathrm{m}$ thick sheets of Kapton and a $1.15-\mathrm{mm}$ thick brass shim; the stack representative of the outer layer consists of 30 insulated conductors covered with five 125 $\mu \mathrm{m}$ thick sheets of Kapton and a $1.2-\mathrm{mm}$ thick brass shim. The conductors are alternated to form a straight stack. Frior to the calibration, the stacks were cured for 3 hours at a temperature of $150 \mathrm{C}$ and a pressure of $100 \mathrm{MPa}$.

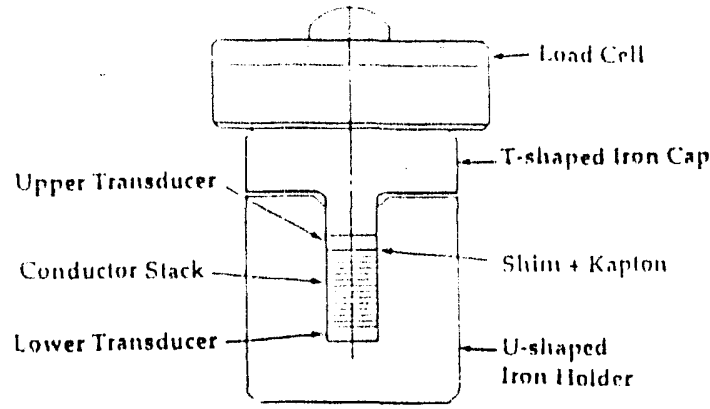

Figure 2: Calibration fixture for beam-type strain gauge transducers.

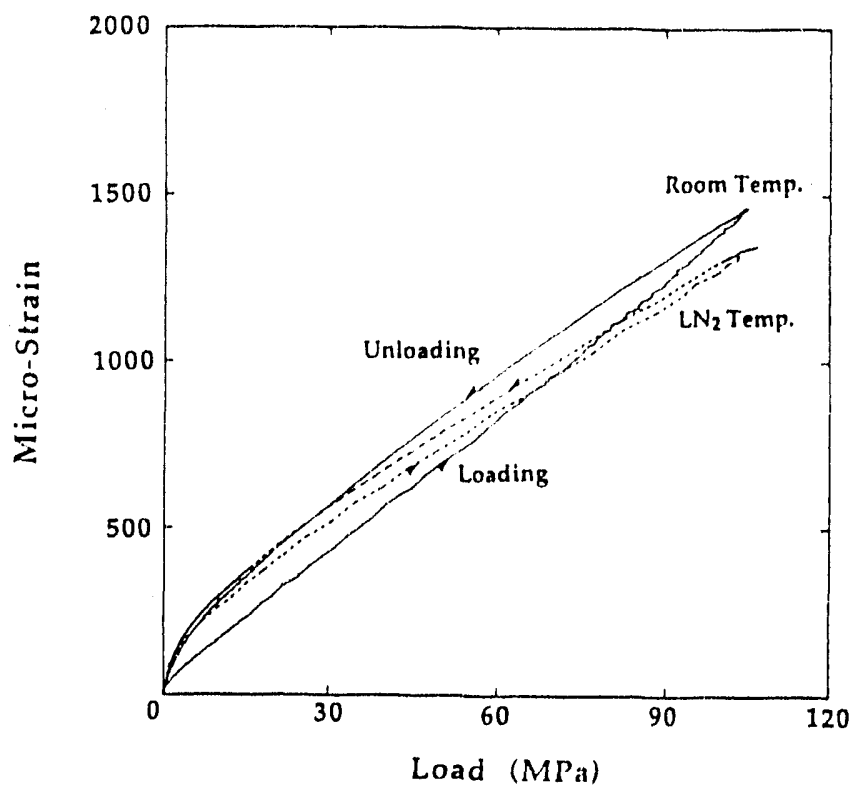

Figure 3: Calijwition curves of beam-type strain gauge transducers.

Figure 2 presents a schematic of the calibration fixture. The conductor stack is inserted inside a U-shaped iron support, with one transducer at the bottom and one at the top, and is covered by a T-shaped iron cap. The load is applied by a hydraulic press on a load cell placed on the top of the T-cap. Finally the whole fixture is placed inside a bucket which can be filled with liquid nitrogen when needed.

Figure 3 shows typical loading/unloading cycles for an inner-conductor stack at room temperature (continuous line) ard at liquid nitrogen temperature (dotted line). The renarkable feature of these curves is the large hysteresis between the loading part of the cycle (bottom) and the unloading part (top). This means that to achieve the same level of strain, a higher stress is required during loading than during unlonding. (Note that the measured strain is not directly the coil deformation, but rather the bending of the beam resulting from that deformation.) The origin of the hysteresis must of course to be located in frictional effects which always oppose deformation: during loading, they oppose compression, thus maintaining a low strain level; during unloading, they oppose release, thus maintaining a high strain level. The next question, then, is to determine where the friction takes place. The most obvious location is against the wall of the stack holder. A simple experiment then consist of switching the top and bottom transducers: if the friction against the wall plays an important role, the readout of a given transducer should be different depending, on its position. In the case of the inner-conductor stack shown in Figure 3, the hysteresis curves are identicai, whether the transducer is on top or on bottom. The friction against the wall is thus negligible, and this hysteresis is an intrinsic property of the conductor stack. 


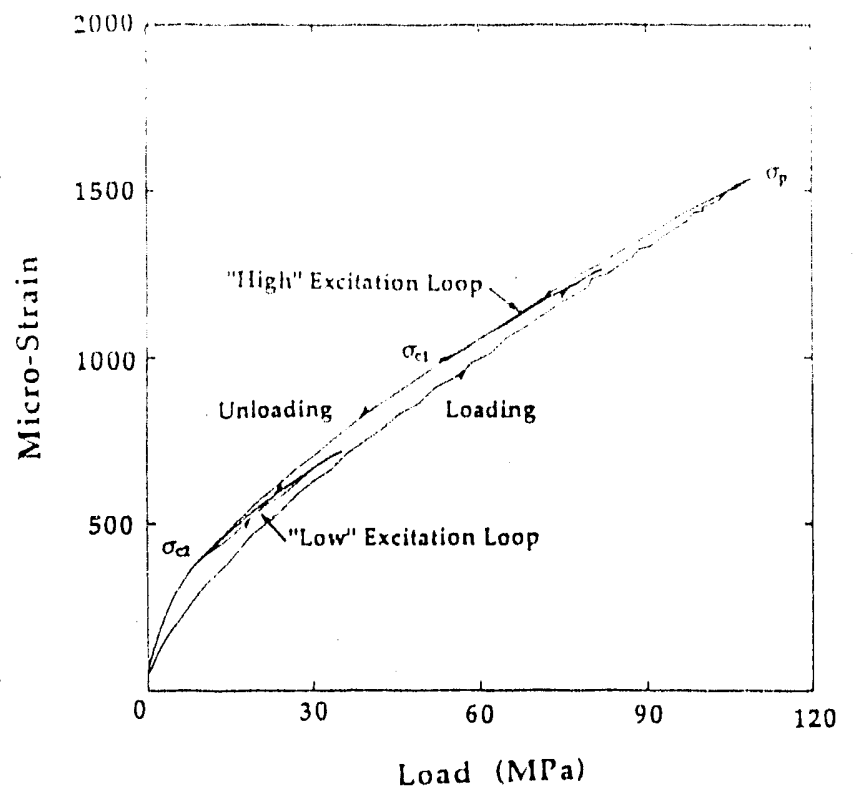

Figure 4: Simulation of coil excitation cycles on a conduclor stack.

Having established that the hysteresis is inherent to the coil, the next step is to examine the consequences of the complicated stress history seen by the coil during the magnet life. Stress histories of dipole coils are presented elsewhere. 8 Schematically, the coil is first loaded at room temperature to a peak stress, $\sigma_{p}$, during collaring. After insertion of the keys, the collaring pressure is relensed, and the coil stress decreases to a value $\sigma_{w}$. The unloading continues throughout cool-down (where the coil shrinks more than the stainless steel collars), and reaches a minimum, $\sigma_{\mathrm{c}}$, at liquid helium temperature. During energization, the coil tends to separate from the collar pole under the Lorentz forces, and the transducer registers an apparent unloading. However, the unloading of the pole corresponds to a compression of the coil; the coil azimuthal stress thus increases to a value $\sigma_{e}$. When the current is ramped down, the coil stress decreases, but since there is hysteresis, it is not clear that the stress goes back to $\sigma_{\mathrm{c}}$. Indeed, an excitation of the coil corresponds to a secondary loop on the main hysteresis, the amplitude of which is not known a priori. To study these secondary loops, we subjected our conductor stacks to a loading/urloading cycle similar to the one we just described. Figure 4 shows an example of strain-gauge readout for an innerconductor stack at liquid-nitrogen temperature (the results are qualitatively the same at $\mathrm{rom}$ temperature). It appears that if the secondary loop is described starting from a high value of $\sigma_{c}$ (that is, if the simulated Lorentz load is applied on a well compacted stack), it lies very close to the unloading part of the main hysteresis, and the behavior of the stack is quasi-elastic. On the other hand, if the secondary loop starts from a low value of $\sigma_{\mathrm{c}}$ (that is, if the simulated Lorentz. load is applied on a not-sowell-compacted stack), it clearly diverges from the main curve, and the return path differs from the loading one: a "spongy" conductor stack thus exhibits a secondary hysteresis. Such ar hysteresis is of course unwelcome during excitation, because it is accompanied by energy dissipation in the coil, and presumably by stick-slip motions of conductors or conduclor strands which are known to cause quenches. ${ }^{9}$ Also, since the coil behavior is inelastic, it may change shape versus time and affect the long-term quality of the high-order multipoles. The conclusion of this experiment is thus that the coil prestress should be chosen so that $\sigma_{\mathrm{c}}$ remains high enough at low temperatures to ensure a quasi-elastic behavior of the coil during excitation.
The results we have discussed so far are purely qualitative. The next step is to figure out how to use the previous curves to calibrate the strain gauges, that is, how to convert the strain readout into actual stress in the coil during the magnet assembly and testing. First of all, the amplitude of the hysteresis depends, on the peak pressure $\sigma_{p}$. The strain gauges should therefore be calibrated for a peak pressure as close as possible to the one which will be seen by the coil during assembly. Next, the collaring data should be converted using the loading part of the calibration curve at room temperature, and the keying data using the unloading part of the same curve. Let $\varepsilon_{w}$ and $\sigma_{w}$ designate the strain and the stress after keying. The next set of data to convert is that of cool-down. The curves in figure 3 show that the strains at low temperature are comparatively lower than those at room temperature, which can be explained by the thermal contraction of the coil stack and the increased stiffness of the stainless steel beam. The question is how to go from one curve to the other. This can be answered by simulating a cooldown on a conductor stack. The same stack as that used in figure 3 was first loaded at room temperature to a peak stress $\sigma_{p}$. The load was then released to a value $\sigma_{w}$, and liquid nitrogen poured into the bucket. Figure 5 presents an example of strain gauge readout during that experiment (the transitory during cool-down is probably due to temperature differences between the two beam gauges and is of no relevance for our analysis). The strains at liquid nitrogen temperature appear to be much higher than those measured when the conductor stack was cooled-down prior to the loading/unlonding cycle as in figure 3 . This again can be explained by frictional effects. In the case of figure 5, the cool-down starts while the stack is alrendy deformed to a level $\varepsilon_{w}$. As the lemperature goes down, friction opposes thermal shrinkage and other effects, maintaining an almost constant level of deformation. Once the stack is cold it then behaves with a Young's modulus very close to that of figure 3. The way to reconcile the two curves of figure 3 is thus to shift the unloading part of the liquid nitrogen calibration along the strain axis until it intersects with the unloading part of the room temperature calibration at $\sigma_{w}$. This allows one to determine $\sigma_{\mathrm{c}}$. The last set of data to convert into stress is that of excitation. This can be done by using the same unloading part of the shifted liquid nitrogen calibration (assuming that $\sigma_{\mathrm{c}}$ is large enough and that there is no secondary hysteresis). All the stresses presented below were converted accordingly.

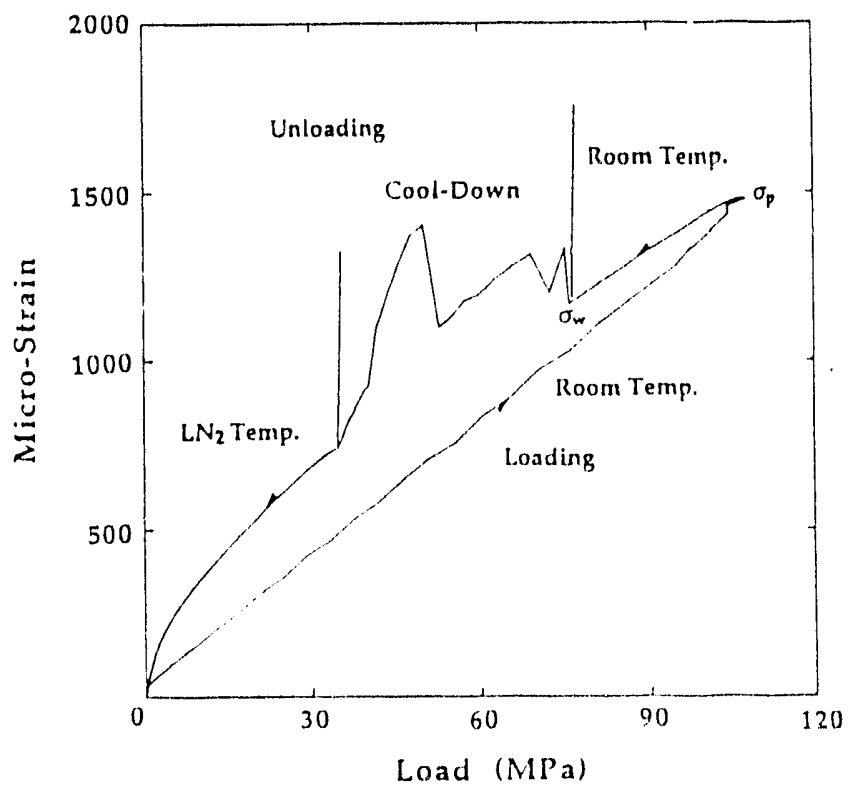

Figure 5: Simulation of cool-down on a conductor stinck. 


\begin{tabular}{|c|c|c|}
\hline & Inner & Outer \\
\hline Poak during collaring ( $\mathrm{MPa}$ ) & 98 & 80 \\
\hline After keying (M!a) & 56 & 50 \\
\hline After yolking ( $(M \Gamma a)$ & 53 & 47 \\
\hline After skinning (MPa) & 55 & 52 \\
\hline Belore cool-down (MPa) & 54 & 52 \\
\hline After cool-down (MPa) & 27 & 44 \\
\hline Stress versus $I^{2}$ slope $\left(M \Gamma a / k \wedge^{2}\right)$ & 0.76 & 0.35 \\
\hline
\end{tabular}

\section{Magnet Assembly and Test}

Table 3 summarizes the stress data recorded during the magnet assembly and test. The discrepancies between the gauge readouts were less than $10 \%$ and the values here reported are averaged over the four inner and outer, gauges.

The peak stress during collaring is comparable to that of the full-length SSC collider dipole prototype DD0017 presented in reference 8 . However, the stress loss after keying reaches 30 $\mathrm{MPa}$, about twice as much as for DD0017. This can probably be explained by differences in the keying technique. in the case of SD501/02, a vertical pressure was applied on the collars until the keyways of the top and bottom collars were aligned to better than $0.4 \mathrm{~mm}$. It is thought that an alignment of $1 \mathrm{~mm}$ would have been sufficient to insert the tapered keys, thus requiring less vertical pressure. The goal is of course to minimize the peak stress seen by the coil during assembly to limit the risk of insulation damage.

A significant difference between SD501/02 and DD0017 appears during the welding of the outer skin: SD501/02 coil stress remains fairly constant, when that of DDO017 increases by more than $10 \mathrm{MPa}$. The explanation for this difference is probably in the mechanics of the collar-yoke interface. Both designs rely on the line-to-line fit concept, that is, the outer radius of the round collars is chosen to be the same as that of the inner radius of the iron yoke. After collaring, because of the pressure exerted by the coil on the cullar pole, the collared-coil assembly becomes oval along the vertical axis. When the yoke is assembled, it pushes on the vertical diameter of the collared coil making it round again, but a small gap eventually remains at the midplane of the yoke. This gap is progressively closed during the welding of the outer skin, and the net result of the yoking and skinning processes is an increase of the coil stress. The amplitude of that increase of course depends on the amount of interference between the collared-coil assembly and the yoke. The collars used for SD501/02 are wider than those of DDO017, and can be estimated to be $55 \%$ stiffer. ${ }^{12}$ They are therefore more difficult to bend, which limits the ovalization of the collaredcoil assembly. If there is less ovalization, there is less interference between the collars and the yoke, and the effect of the skin welding on the coil stress should therefore be limited, as observed on the strain gauges. Partial measurements of the collared-coil assembly vertical and horizontal diameters, which appear to be less or equal to the maximum design values of the inner diameters of the yoke, support this explanation, as does a visual observation that the gap at the midplane of the yoke was already closed before beginning the shell welding. All these elements therefore rombine to deinonstrate that SD501/02 has a weak collar-yoke interference.

The stress loss during cool-down of the inner layer is similar to that of the inner layer of DD0017, but the stress loss of the outer layer is only half that measured on DD0017. This differ. ence can probably be attributed to the difference in geometry of the outer layers, which do not have the same angles. Also, the surface of the collar pole against which the outer layer of DD0017 presses is not radial.

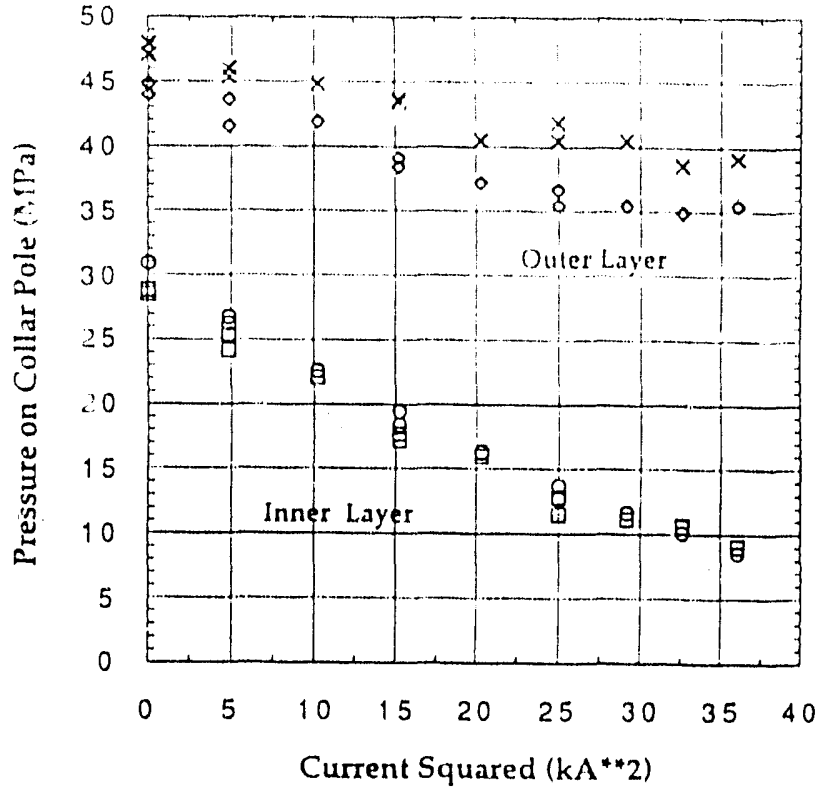

Figure 6: Variation of the average pressure exertec $y$ the coil on the coilar pole of magnet SD501/02 during excitation.

Figure 6 shows an example of stress variation during excitation. Both inner- and outer- layer stresses start by decreasing linearly versus the current squared, as expected from the Lorentz forces. The decrease slows down at higher currents, but doee not flatten as seen on recent SSC prototypes, ${ }^{8}$ indicating that the coil does not separate from the collar pole. Furthermore, there is no indication of hysteresis when ramping down the current, which tells us that the coil is operated at a high enough level of stress, according to the criterion presented in the previous paragraph. Table 3 presents the average slope of stress versus the current squared curves at low excitation; it is similar to that of DD0017 for the inner layer, but the outer-layer slope is much larger (by a factor 2.5). Once again, this difference can probably be attributed to the difference in geometry of the outer layers.

Table 4. Quench Summary of Maģnel SD501/02

\begin{tabular}{llll} 
& & & \\
& Current $(\Lambda)$ & Field $(\mathrm{T})$ & Location \\
\hline Quench 1 & 5157 & 5.77 & Lower outer \\
Quench 2 & 5797 & 6.51 & Upper inner \\
Quench 3 & 6160 & 6.85 & (no data) \\
Quench 4 & 6495 & 7.17 & Lower splice \\
Quench 5 & 6602 & 7.29 & Upper outer \\
Quench 6 & 6668 & 7.33 & Upper inner pole lurn \\
Quench 7 & 6820 & 7.48 & Lower inner pole tum \\
Quench 8 & 6815 & 7.48 & Lower inner pole lum \\
\hline
\end{tabular}

Table 4 summarizes the quench perfornance of SD501/02. It took two quenches to reach the design current $(5968 \mathrm{\Lambda})$, and four more quenches to reach a plateau of $6820 \mathrm{~A}$. Most of the training quenches originated in the outer layer, which includes the splice area, but has no voltage tap. (The splice between the inner- and outer-layers is made at the beginning of the outerlayer pole tum by ramping up the conductor of the inner-layer pole turn. A voltage tap is located between the ramp and splice sections which delimits the inner and outer quarter-coil voltages.) It is clear from the voltage traces of quench 4 , that the quench began very close to the quarter tap, on the splice side. The delay in reaching the quarter tap is much longer for quenches 1 and 5 , but the propagation along the ramp section is also much slower; there is therefore some suspicion that these 
iwo quenches also originated in the splice. The serpuence of yolages for quench 2 is not entirely consistent, which prohibils a detailed analysis, but it also oniginated in the tamp-splice area As for the last three quenches, they all occurred in the pole turn of the inner layer, near the magnet center.

From the data we presented. Iwo explanations can be found of the relatively poor traning behavior of the magnet. One is a bad splicing technique (note that both the lower and upper splices are in question). A second is the weak interference between the collars and the yoke, which does not provide good enough radial support to the coil during excitation. There is lit lle that can be done to lix the spitices, except taking the magnet apart. The interference problem can be more easily fixed: it consists by removing the outer skin and the yoke, and by putting a brass shim on the outer surface of the collars, as was done on earlier full-length SSC dipole prototypes. ${ }^{13}$

\section{Conclusion}

Experiments on the conductor stacks were carried out in order to understand the mechanical properties of a magnet coil. They pointed out the existence of important frictional effects resulting in the hysteretic behavior of a loading/unloading cycle. They also showed that the hysteresis during excitation was greatly reduced if the coil stress at liquid helium temperature was maintained at a high level. A magnet was built following these principles, which however exhibited relatively poor training behavior. This poor training can be attributed to a bad splicing technique, a lack of stiflness in the coil radial support, or a combination of the two.

\section{Acknowledgements}

The authors are indebted to Prof. H Sugawara and Prof. $Y$ Kimura for their continuous support. They wish to thank the staff of the KEK Mechanical Engineering and Cryogenic Centers for their dedication and hard work. They are also grateful to Giancarlo Spigo for his Insightful comments, and to Jared Stark for his help in editing the text.

\section{REFERENCES}

11) Conceptual Design of the Supercunducting Super Gollider, I. D. Jackson, ed., SSC-SR-2020, warth 1986, reviced, Seplemter 1988.

121 The Laree Hadron Collider in the LEL Lumel (j. Brianti and $\mathrm{K}$. Hübner, eds, CERN 87-(15, May 1, 1987.

|3| T. Shintomi, el al, "Development of Large Keystone Angle Cable for Dipole Magnet with !deal Arch Structure," Adu Cryo. Eng. Malerials, 36^, fp. 323-328, 1900 .

[4] H. li, el al, "Trial-fabrication of Superconducting Rutherford Type (able Having, Large Kyestone Angle," Proccedings ol the 11/h

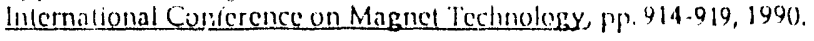

15) T. Shinlomi, et al., "Superconducting Dipole Magnet with Large Keystone Angle Calle for High Energy I Hadron Accelerators," Particle Ascelcrator 28, pp. 219-224, 1990.

(6) T. Shintomi, et al., "Development of Superconducting Dipole Magne't with Ideal Arch Struclure Using Large Keystone Angle Cable," Proceedings of the 11 th International Conference en Magnes Technology pp. 181-186, 1990.

(7) P. Dahl, et al., "Construction of Cold Mass Assembly for Full Length Dipoles for the SSC Accelerator," IEEE Transaction on Magnctics, 23, pp. 1215-1218, March 1987.

(8) A. Devred, et al." Test Results of Full-Length SSC Collider Dipele Prototypes," these proceedings.

$19 \mid \wedge$. Devred, "Quench Origins," to appear in the proceedings of 1989/1990 US Particle Accelerator School, Upton, NY, USA, July 29-August 4, 1990.

|10| $\Lambda$. Devred, et al., "Quench Start Localization in Full-length SSC R\&D Dipoles," Supercollider 1, M. McAshan, ed., pp. 73-83, 1989.

111] C. Goodzeit, et al., "Mcasurement of Internal Forces in Superconducting Accelerator Magnets with Strain Gauge. Transducers," IEEE Transactions on Magnclics, 25, pp. 1463-1468, March 1989.

1121 G. Spigo, private communication.

(13)1. Tompkins, el al., "Performance of Full-Lenglh SSC Model Dipoles: Results from 1988 Tests," Supercollider 1, M. McAshan, ed., pp. 33-49, 1989. 

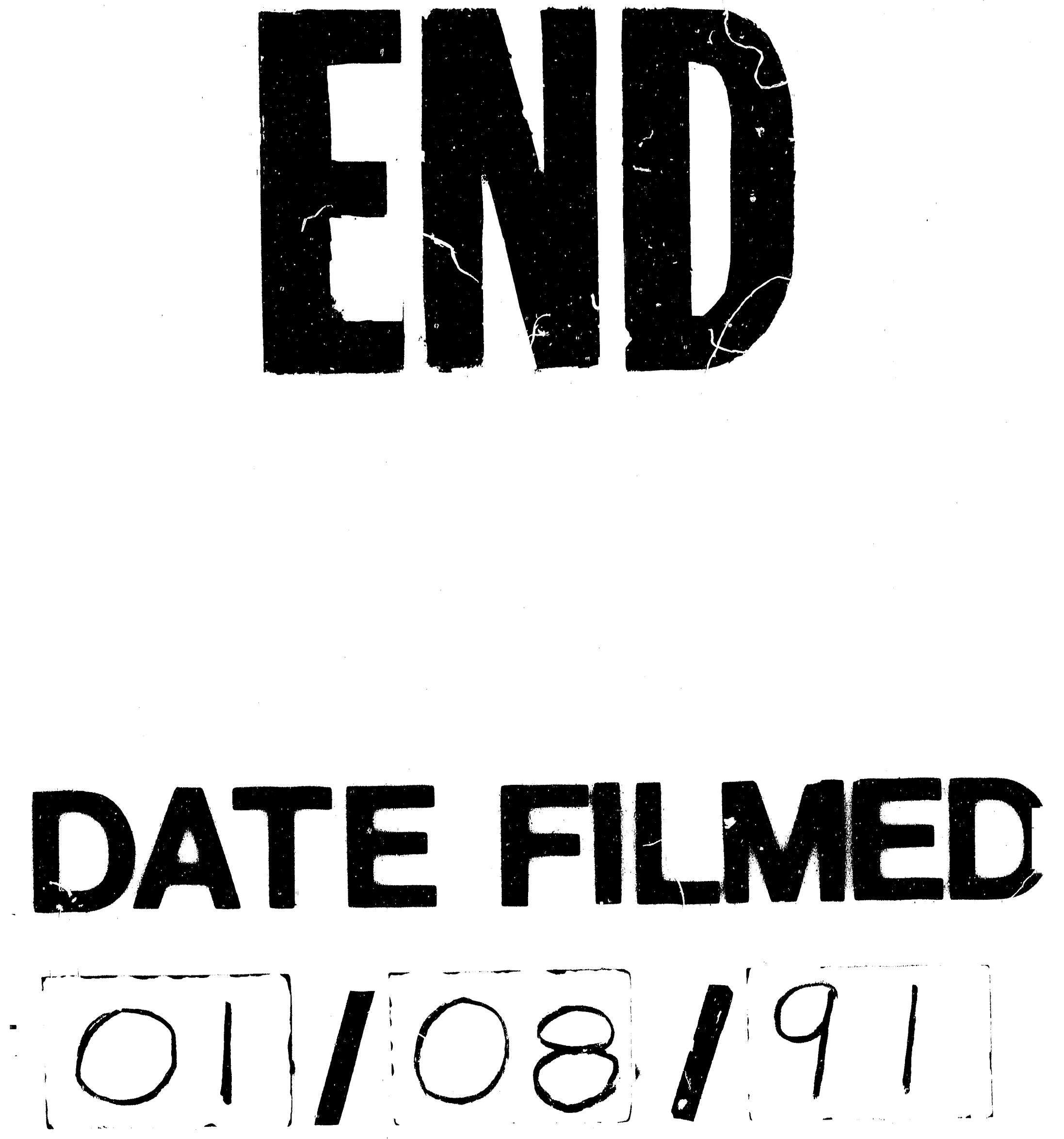
